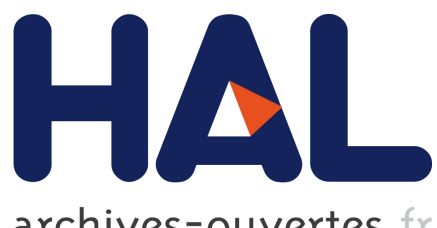

archives-ouvertes

\title{
Bouncing of a dispersive wave in a solitonic cage
}

\author{
S. Wang, A. Mussot, M. Conforti, X. Zeng, A. Kudlinski
}

\section{To cite this version:}

S. Wang, A. Mussot, M. Conforti, X. Zeng, A. Kudlinski. Bouncing of a dispersive wave in a solitonic cage. Optics Letters, Optical Society of America, 2015, 40 (14), pp.3320. 10.1364/OL.40.003320 . hal-02389000

\section{HAL Id: hal-02389000 \\ https://hal.archives-ouvertes.fr/hal-02389000}

Submitted on 2 Dec 2019

HAL is a multi-disciplinary open access archive for the deposit and dissemination of scientific research documents, whether they are published or not. The documents may come from teaching and research institutions in France or abroad, or from public or private research centers.
L'archive ouverte pluridisciplinaire HAL, est destinée au dépôt et à la diffusion de documents scientifiques de niveau recherche, publiés ou non, émanant des établissements d'enseignement et de recherche français ou étrangers, des laboratoires publics ou privés. 


\title{
Bouncing of a dispersive wave in a solitonic cage
}

\author{
S. F. WANG ${ }^{1,2}$, A. Mussot ${ }^{1}$, M. Conforti ${ }^{1}$, X. L. ZenG ${ }^{2}$, AND A. Kudlinski ${ }^{1, *}$ \\ ${ }^{1}$ Laboratoire PhLAM, UMR CNRS 8523, IRCICA, USR CNRS 3380, Université Lille 1, 59655 Villeneuve d'Ascq, France \\ ${ }^{2}$ The Key Lab of Specialty Fiber Optics and Optical Access Network, Shanghai University, 200072 Shanghai, China \\ *Corresponding author: alexandre.kudlinski@univ-lille1.fr
}

Compiled June 23, 2015

\begin{abstract}
We report the experimental observation of a weak dispersive wave trapping within a cage formed by two solitons in an optical fiber. We show that the dispersive wave bouncing is accompanied by a back and forth wavelength conversion of the probe to an idler wave. Besides, we observed the destruction of the soliton cage when dispersive wave power is increased, leading to the collision of the solitons.
\end{abstract}

OCIS codes: (190.4370) Nonlinear optics, fibers; (190.5530) Pulse propagation and temporal solitons.

http://dx.doi.org/10.1364/ol.XX.XXXXXX

The nonlinear interaction of a soliton with a weak dispersive wave (DW) in optical fibers has been the subject of intense investigations over the past ten years, due to its key role in explaining the process of supercontinuum (SC) generation [1]. In this context, theoretical and experimental studies reviewed in Ref. [1] showed the generation of new frequency components due to four-wave mixing (FWM) between solitons and DWs [24] during their collision. In the time domain, the physics of the reflection of a weak DW on a soliton led to analogies with the emission of Hawking radiation at the event horizon of a black hole [5] or with the superfluidity of light [6].

Increasing the DW power with respect to the soliton one has the effect of modifying the soliton characteristics through crossphase modulation [7-11], opening the way to all-optical signal processing applications [10]. Recently, studies of soliton-DW dynamics were pushed one step further by considering the interaction of a DW with two identical time-separated solitons [12]. It was shown numerically that a DW may experience multiple reflections between the two solitons, causing a stepwise frequency conversion of the DW due to FWM with the colliding soliton. If the DW is powerful enough, the soliton properties are slightly modified after each collision with the DW, which changes their velocity and eventually leads to their collision. This effect has been numerically shown to affect the spectral characteristics of SC spectra under specific conditions [13].

In the present work, we experimentally observe the bouncing of a DW between a pair of identical solitons, through the measurement of the back and forth frequency conversion of the DW at each soliton reflection. Furthermore, we show that the adjustment of the DW power can be used to control the delay between the two solitons and induce their collision, adding a new degree of freedom to the field of all-optical signal processing.

Figure 1 shows the experimental setup built in order to observe the process of DW bouncing. A Ti:Sa oscillator delivers gaussian pulses with a full-width at half maximum (FWHM) duration of $140 \mathrm{fs}$ around $800 \mathrm{~nm}$ at $80 \mathrm{MHz}$. After a beam splitter, one beam (depicted in blue in Fig. 1) serves as the probe wave and the other one is used to pump an optical parametric oscillator (OPO). A system made of a polarizer placed between two half-wave plates allows to adjust the polarization state and power of the probe. Then a $0.3 \mathrm{~nm}$ FWHM bandpass filter centered at $802 \mathrm{~nm}$ is used to temporally broaden the probe. The pulses coming out of the OPO (depicted in red in Fig. 1) have a gaussian shape, a FWHM duration of $250 \mathrm{fs}$ and can be tuned between 1000 and $1500 \mathrm{~nm}$. They will be termed as pump pulses hereafter and will be used to generate the solitonic cage in the fiber. To do that, we use the architecture of a Michelson interferometer. One arm has a moving mirror mounted on a micrometer screw, in order to control the temporal delay between the two pulses. Each arm contains a quarter-wave plate to control the polarization state, resulting in an independent power control of each pulse after the half-wave plate and polarizer placed at the output. A final half-wave plate is used to align the polarization state of this double pulse with the one of the probe as well as with one birefringent axis of the fiber. The delay between the two pump pulses is carefully measured using

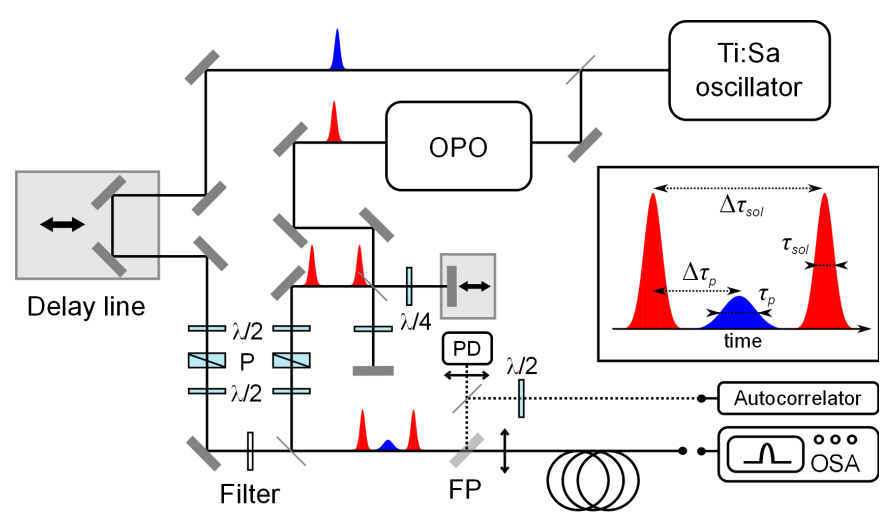

Fig. 1. Experimental setup. P: polarizer; FP: flipping mirror; PD: photodiode. 
an autocorrelator. The delay of the probe relatively to this double pulse is adjusted with a delay line placed on the probe path and is measured using two-photon cross-correlation in a photodiode [14]. The inset of Fig. 1 shows a schematic representation of the available pulses, where $\tau_{p}$ is the probe duration, $\tau_{s o l}$ is the pump duration, $\Delta \tau_{p}$ is the delay between the probe and the first pump pulse, $\Delta \tau_{\text {sol }}$ is the delay between the two pump pulses, both of which can be carefully adjusted. Additionally, the polarization state and power of all three pulses can be adjusted simultaneously and independently.

These pulses are then coupled into a $29 \mathrm{~m}$-long photonic crystal fiber (PCF) with a pitch $\Lambda$ of about $2.9 \mu \mathrm{m}$ and a relative hole diameter $d / \Lambda$ of about 0.58 . The simulated PCF zero dispersion wavelength (ZDW) is $980 \mathrm{~nm}$ and the nonlinear parameter at the pump and probe wavelength are estimated about $15 \mathrm{~W}^{-1} \cdot \mathrm{km}^{-1}$ and $24 \mathrm{~W}^{-1} \cdot \mathrm{km}^{-1}$, respectively. In order to induce collisions between the double soliton and the probe, both radiations have to be slightly off the group velocity (GV) matching condition. Since the probe has a fixed wavelength of $802 \mathrm{~nm}$ (imposed by the bandpass filter), the wavelength of the double pulse exciting the double soliton has to be determined from the dispersion properties of the PCF. The black curve in Fig. 2(a) shows the phase-matching (PM) curve (calculated from the PCF properties) associated to the FWM process between the probe located at $802 \mathrm{~nm}$ (blue marker) and a soliton located at $1210 \mathrm{~nm}$ (red marker) during their collision. It can be seen graphically that the conversion of the probe into an idler wave located around $792.5 \mathrm{~nm}$ [green marker in Fig. 2(a)] is expected $[2,15]$.

The spectrum and autocorrelation trace of the incident probe located at $802 \mathrm{~nm}$ are shown in Fig. 2(b) and (c) respectively. The spectrum is slightly asymmetric due to the shape of the bandpass filter, and the resulting pulse is well fitted by a lorentzian curve with a FWHM duration $\tau_{p}$ of $600 \mathrm{fs}$. Figures 2(d) and (e) show respectively the spectrum and autocorrelation trace of the double pulse at the PCF input. The spectrum exhibits spectral fringes with a very good contrast, which is op-
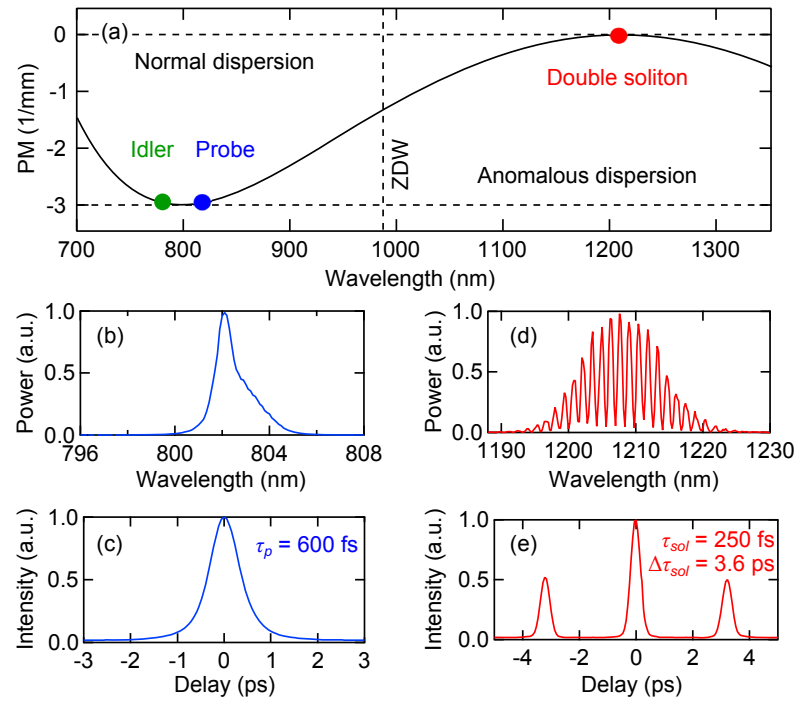

Fig. 2. (a) PM curve calculated from the fiber parameters given in the text. (b) Measured spectrum and (c) autocorrelation trace of the probe at the fiber input. (d) Measured spectrum and (e) autocorrelation trace of the double pump pulse at the fiber input. timized to ensure that the two pulses have identical power. This is confirmed by the overall shape of the autocorrelation trace, in which the side lobes intensity reaches half the one of the main peak. The measured duration of each pulse is $\tau_{s}=250 \mathrm{fs}$ and their relative delay is $\Delta \tau_{s o l}=3.6 \mathrm{ps}$. Now, by ensuring that the probe is located temporally in between the two $1210 \mathrm{~nm}$ pulses (using the cross-correlation measurement described above), we will focus our investigation to the observation of the multiple reflection scenario presented in [12].

Before performing experiments, we checked numerically that our experimental parameters indeed allow to observe the trapping of the DW in the solitonic cage. We used the generalized nonlinear Schrödinger equation (GNLSE) including the full dispersion curve, the frequency dependent nonlinear parameter, as well as Kerr (including the shock term) and Raman nonlinearities. The temporal evolution versus fiber length is depicted in Fig. 3(a), where two gaussian pulses (peak power of 75 $\mathrm{W}$, FWHM duration of $250 \mathrm{fs}$ ) and a weak probe (peak power of $1.5 \mathrm{~W}, \mathrm{FWHM}$ duration of $600 \mathrm{fs}$ ) are simultaneously launched into the PCF. The relative GV difference induces the collision of the probe with the first soliton. Remarkably, the probe is almost completely reflected after the first collision process. It then goes towards the second soliton and the same collision process occurs again. Three collisions are observed along the $29 \mathrm{~m}$-long fiber. At the fiber output, the soliton separation ( $3 \mathrm{ps}$ ) is slightly reduced as compared to the fiber input (3.6 ps), as a result of the weak (but non negligible) nonlinear interaction between the probe and solitons at each collision. Figure 3(d) shows the corresponding spectral dynamics. As expected, the probe initially located at $802 \mathrm{~nm}$ is totally converted into an idler located at $792.5 \mathrm{~nm}$ during the first collision, which is consistent with the interpretation of Fig. 2(a). After the next collision, the probe reversely converts to $800 \mathrm{~nm}$, which is slightly shorter than the incident wavelength. The reason is that the PM relation is modified in view of the fact that two solitons slightly red-shift to longer wavelengths owing to the Raman effect. This wavelength conversion occurs at each collision between the probe and one of the solitons, and is thus an unambiguous signature of the trapping of the probe within the solitonic cage.

Based on these realistic simulation results, the corresponding experiment was performed. In the following, all parameters (except the probe peak power) are fixed and equal to those of simulations. Firstly, we equalized the relative power of the two solitons by observing their autocorrelation trace and simultaneously maximizing the contrast of their spectral fringes, both at the PCF output. The corresponding measurements are shown in Figs. 3(b) and (c) with red markers and red line respectively, together with the corresponding numerical simulations in black lines. They are both in good agreement, which indicates that the experimental configuration is very close to the simulation results of Figs. 3(a) and (d). More precisely, two symmetric side lobes with half intensity of the main peak of the autocorrelation trace are observed, suggesting that two nearly identical solitons are still sustained at the fiber output. Moreover, the resultant delay between two solitons is around 3 ps, indicating that two solitons are slightly reshaped due to the interaction with the probe, as observed in simulations. Finally, the output spectra of the two solitons shown in Fig. 3(c) also exhibit a slight Raman red shift. All these observations are in excellent agreement with numerical simulations, which indicates that the experimental configuration is very close to the one used in previous simulations. In order to get a deeper insight about the dynamics versus fiber length, we recorded the spectral evolution using a cutback ap- 

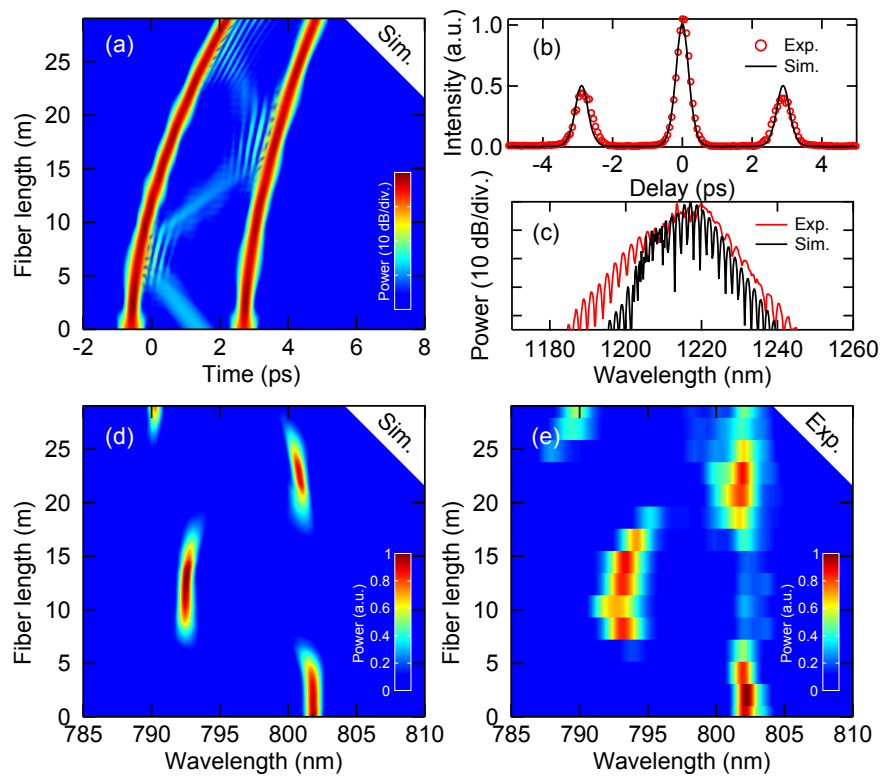

Fig. 3. (a) Simulated temporal evolution versus fiber length. (b) Measured (red open circles) and simulated (black solid line) soliton autocorrelation traces at the fiber output. (c) Measured (red solid line) and simulated (black solid line) output spectra of the double soliton. (d) Simulated and (e) measured spectral dynamics in the probe region.

proach, in which the spectrum is recorded after successive fiber cuts of $2 \mathrm{~m}$. The result is depicted in Fig. 3(e) and is in excellent agreement with the corresponding simulation of Fig. 3(d). It remarkably illustrates the cascaded wavelength conversions of the probe to the idler (and vice versa) across the GV matching wavelength, which is the signature of the multiple reflections of the probe between the two solitons. These results constitute the first experimental demonstration of the trapping of a dispersive wave in a solitonic cage.

We now further explore the influence of the probe power on the solitonic cage, as it is known that the soliton characteristics can be affected by the collision through cross-phase modulation when the probe has a high enough power [8, 10, 11]. While simulations and experiments of Fig. 3 were performed for a probe peak power of $1.5 \mathrm{~W}$, Figs. 4 (a) and (d) show numerical simulations of the temporal traces for higher probe peak powers of respectively 5.2 and $9.2 \mathrm{~W}$. Although in both cases the probe remains well confined within the solitonic cage during the first two collisions, these two interactions modify both the soliton and probe characteristics, as demonstrated in earlier papers $[8,10,11]$. As a result, in the next (third) collision, the probe cannot be totally reflected and a significant fraction of its energy eventually escapes from the solitonic cage. The consequence of the nonlinear interaction of the probe and solitons is that both solitons now have significantly different characteristics so that they collide after the probe has escaped. In these two examples, the collision occurs at the fiber output for the lower probe power of $5.2 \mathrm{~W}$ [Fig. 4(a)] and around $22 \mathrm{~m}$ for the probe power of $9.2 \mathrm{~W}$ [Fig. 4(b)]. Of course, this modified picture of the temporal dynamics changes the spectral dynamics as well, as shown in Figs. 4(c) and (f), respectively. In both cases, the almost complete stepwise wavelength conversion between the probe and the idler is observed during their first two collisions, but the modified dynamics of the third collision results
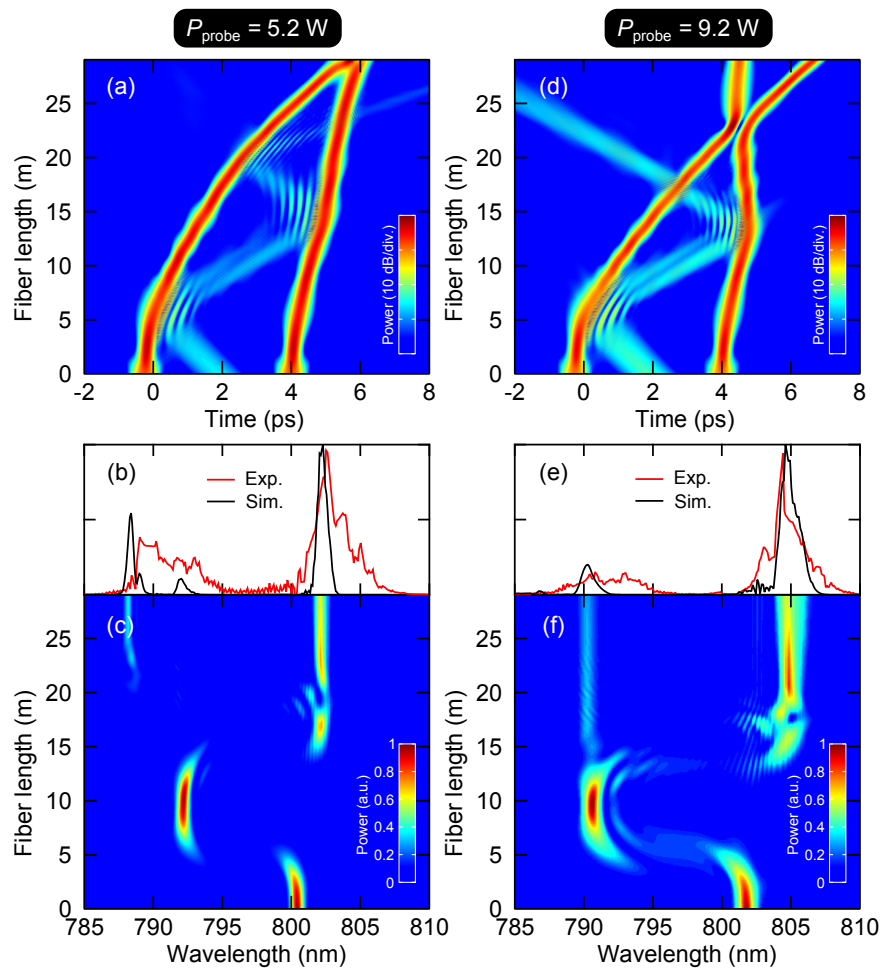

Fig. 4. (a) Simulated temporal evolution versus fiber length for a probe peak power of $5.2 \mathrm{~W}$. (b) Measured (red line) and simulated (black line) output spectrum in the probe spectral region. (c) Simulated spectral dynamics of the probe versus fiber length. (d)-(f) Same representation for a probe peak power of $9.2 \mathrm{~W}$.

in a significantly modified energy exchange between the probe and idler. As a result, the output spectra shown in Figs. 4(b) and (e) exhibit two peaks corresponding to the probe (beyond $800 \mathrm{~nm}$ ) and the idler (below $800 \mathrm{~nm}$ ). The output conversion efficiency into the idler depends on the probe peak power, as confirmed experimentally [red curves in 4(b) and (e)] in good agreement with simulations [black curves in 4(b) and (e)]. This is an unambiguous signature of a strong nonlinear interaction between the probe and the solitons.

In order to characterize more accurately this phenomenon, we numerically and experimentally investigated their delay as a function of probe peak power. Figure 5 shows the simulation result in solid black line and experimental results depicted by markers, which are in good agreement. Experimentally, the delay between the two solitons was deduced from their autocorrelation trace measured at the fiber output, represented in the insets of Fig. 5. The green curve corresponds to the case of Fig. 3 while the red and blue ones correspond to the cases of Fig. 4(b) and (e), respectively. Black dots depict other measurements performed but not shown here for the sake of clarity. As we could infer from the previous results, the output soliton delay firstly decreases with increasing probe power, until the two solitons collide and thus merge for a probe power around $5 \mathrm{~W}$. In this region, depicted by the grey area, it is not possible to evaluate the exact delay between the two solitons since they merge, as illustrated by the experimental autocorrelation trace plotted in red. By further increasing the probe power, the output soliton delay increases again, as the collision occurs at a shorter fiber length, as illustrated in Figs. 4(a) and (d). For the particular example 


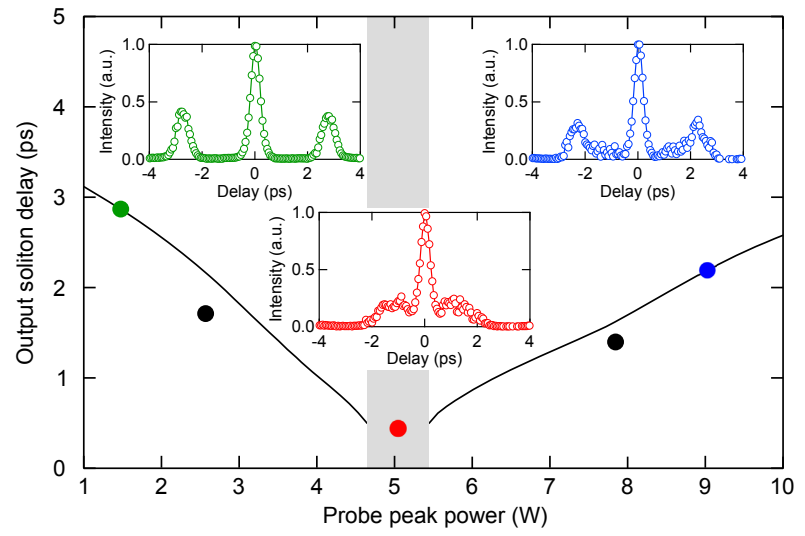

Fig. 5. Simulated evolution of the delay between the two solitons at the output of the $29 \mathrm{~m}$-long fiber as a function of probe peak power. Markers correspond to measurements from autocorrelation traces. Insets are examples of experimental autocorrelation traces for probe peak powers of $1.5 \mathrm{~W}$ (left, green), $5.2 \mathrm{~W}$ (middle, red) and 9.2 W (right, blue). The grey shaded area corresponds to the region in which the two solitons collide and therefore cannot be distinguished.

depicted in blue in Fig. 5 (corresponding to a probe power of $9.2 \mathrm{~W}$ ), the output autocorrelation trace exhibits two side lobes with an intensity which is much less than half the main peak intensity, which indicates that the two individual solitons have different intensities. This is due to the fact that the interaction with the probe (which is about tenfold smaller than each soliton in this case) has modified their characteristics at each collision, and also to the fact that the collision between the two solitons is not perfectly elastic.

In summary, we have experimentally observed the trapping of a dispersive wave within a solitonic cage made of two identical solitons. A careful adjustment of the probe power allows to shape the temporal trajectory of the two solitons. We show experimentally that this effect can be used to induce their collision at a desired location within the fiber, and to control their relative delay at the fiber output. Besides their fundamental interest, these results constitute a further step towards all-optical ultrafast signal processing.

This work was partly supported by the ANR TOPWAVE (ANR-13-JS04-0004) and NoAWE (ANR-14-ACHN0014) projects, and by the "Fonds Européen de Développement Economique Régional", the Labex CEMPI (ANR-11-LABX0007) and Equipex FLUX (ANR-11-EQPX-0017) through the "Programme Investissements d'Avenir". S. F. Wang acknowledges the grant of the China Scholarship Council. X. L. Zeng acknowledges the research support by National Natural Science Foundation of China (11274224) and the Program for Professor of Special Appointment (Eastern Scholar) at Shanghai Institutions of Higher Learning.

\section{REFERENCES}

1. D. V. Skryabin and A. V. Gorbach, Rev. Mod. Phys. 82, 1287 (2010).

2. A. V. Yulin, D. V. Skryabin, and P. St. J. Russell, Opt. Lett. 29, 2411 (2004).

3. D. V. Skryabin and A. V. Yulin, Phys. Rev. E 72, 016619 (2005).

4. A. Efimov, A. V. Yulin, D. V. Skryabin, J. C. Knight, N. Joly, F. G. Omenetto, A. J. Taylor, and P.St.J. Russell, Phys. Rev. Lett. 95, 213902 (2005).
5. T. G. Philbin, C. Kuklewicz, S. Robertson, S. Hill, F. König, and U. Leonhardt, Science 319, 1367 (2008).

6. S. Batz and U. Peschel, Phys. Rev. A 85, 033824 (2012).

7. W. H. Loh, A. B. Grudinin, V. V. Afanasjev, and D. N. Payne, Opt. Lett. 19, 698 (1994)

8. V. E. Lobanov and A. P. Sukhorukov, Phys. Rev. A 82, 033809 (2010).

9. R. Driben, F. Mitschke and N. Zhavoronkov, Opt. Express 18, 25993 (2010).

10. A. Demircan, S. Amiranashvili, and G. Steinmeyer, Phys. Rev. Lett. 106, 163901 (2011).

11. L. Tartara, J. Opt. Soc. Am. B 32, 395 (2015).

12. A. V. Yulin, R. Driben, B. A. Malomed, and D. V. Skryabin, Opt. Express 21, 14481 (2013).

13. R. Driben, A. V. Yulin, A. Efimov, and B. A. Malomed, Opt. Express 21, 19091 (2013).

14. C.-H. Hage, F. Billard, B. Kibler, C. Finot and G. Millot, Electron. Lett. 48, 778 (2012).

15. K. E. Webb, M. Erkintalo, Y. Xu, N. G. R. Broderick, J. M. Dudley, G. Genty, and S. G. Murdoch, Nat. Commun. 5 (2014). 


\section{INFORMATIONAL FIFTH PAGE}

\section{REFERENCES}

1. D. V. Skryabin and A. V. Gorbach, "Colloquium : Looking at a soliton through the prism of optical supercontinuum," Rev. Mod. Phys. 82, 1287-1299 (2010).

2. A. V. Yulin, D. V. Skryabin, and P. St. J. Russell, "Four-wave mixing of linear waves and solitons in fibers with higher-orderdispersion," Opt. Lett. 29, 2411-2413 (2004).

3. D. V. Skryabin and A. V. Yulin, "Theory of generation of new frequencies by mixing of solitons and dispersive waves in optical fibers," Phys. Rev. E 72, 016619 (2005).

4. A. Efimov, A. V. Yulin, D. V. Skryabin, J. C. Knight, N. Joly, F. G. Omenetto, A. J. Taylor, and P.St.J. Russell, "Interaction of an Optical Soliton with a Dispersive Wave," Phys. Rev. Lett. 95, 213902 (2005).

5. T. G. Philbin, C. Kuklewicz, S. Robertson, S. Hill, F. König, and U. Leonhardt, "Fiber-Optical Analog of the Event Horizon," Science 319, 13671370 (2008).

6. S. Batz and U. Peschel, "Superfluid motion of light past a solitonic obstacle," Phys. Rev. A 85, 033824 (2012).

7. W. H. Loh, A. B. Grudinin, V. V. Afanasjev, and D. N. Payne, "Soliton interaction in the presence of a weak nonsoliton component," Opt. Lett. 19, 698-700 (1994).

8. V. E. Lobanov and A. P. Sukhorukov, "Total reflection, frequency, and velocity tuning in optical pulse collision in nonlinear dispersive media," Phys. Rev. A 82, 033809 (2010).

9. R. Driben, F. Mitschke and N. Zhavoronkov, "Cascaded interactions between Raman induced solitons and dispersive waves in photonic crystal fibers at the advanced stage of supercontinuum generation", Opt. Express 18, 25993-25998 (2010).

10. A. Demircan, S. Amiranashvili, and G. Steinmeyer, "Controlling Light by Light with an Optical Event Horizon," Phys. Rev. Lett. 106, 163901 (2011).

11. L. Tartara, "Soliton control by a weak dispersive pulse," J. Opt. Soc. Am. B 32, 395-399 (2015).

12. A. V. Yulin, R. Driben, B. A. Malomed, and D. V. Skryabin, "Soliton interaction mediated by cascaded four wave mixing with dispersive waves," Opt. Express 21, 14481-14486 (2013).

13. R. Driben, A. V. Yulin, A. Efimov, and B. A. Malomed, "Trapping of light in solitonic cavities and its role in the supercontinuum generation," Opt. Express 21, 19091-19096 (2013).

14. C.-H. Hage, F. Billard, B. Kibler, C. Finot and G. Millot, "Direct temporal reconstruction of picosecond pulse by cross-correlation in semiconductor device," Electron. Lett. 48, 778-779 (2012).

15. K. E. Webb, M. Erkintalo, Y. Xu, N. G. R. Broderick, J. M. Dudley, G. Genty, and S. G. Murdoch, "Nonlinear optics of fibre event horizons," Nat. Commun. 5 (2014). 